\title{
A New Linearly Combined Nyquist Pulses for PAPR Reduction in IFDMA
}

\author{
Amina Mohammad \\ Electronics and Communications \\ Dept. \\ Modern Academy for Engineering \\ and Technology, Cairo, Egypt
}

\author{
Bassant Abdelhamid \\ Electronics and Communications \\ Dept. \\ AinShams University, \\ Cairo, Egypt
}

\author{
I.M. Hafez \\ Electronics and Communications \\ Dept. \\ AinShams University, \\ Cairo, Egypt
}

\begin{abstract}
Single Carrier Frequency Division Multiple Access (SCFDMA) is proposed for uplink transmission of Long Term Evolution Advanced (LTE-A) due to its lower Peak to Average Power Ratio (PAPR) compared to Orthogonal Frequency Division Multiple Access (OFDMA). In this paper, a comparison is introduced between its two subcarrier mapping schemes, Localized-FDMA (LFDMA) and Interleaved-FDMA (IFDMA), in terms of PAPR. Moreover, the paper addresses the performance of SC-FDMA with Pulse Shaping (PS). Since conventional Raised Cosine (RC) PS introduces a trade-off between spectrum efficiency and low PAPR in IFDMA, the results show that some newly combined Nyquist pulses can reduce PAPR of IFDMA significantly while maintaining the same Bandwidth (BW) compared to conventional RC pulse.
\end{abstract}

\section{Keywords}

Single Carrier Frequency Division Multiple Access (SCFDMA), Long Term Evolution Advanced (LTE-A), Peak-toAverage Power Ratio (PAPR), Localized-FDMA (LFDMA), Interleaved-FDMA (IFDMA), Raised Cosine (RC).

\section{INTRODUCTION}

In the Third Generation Partnership Project (3GPP) Long Term Evolution (LTE) and LTE-Advanced (LTE-A) standards, the technologies being implemented consist of Single Carrier Frequency Division Multiple Access (SCFDMA) for uplink and Orthogonal Frequency Division Multiple Access (OFDMA) for downlink. Both of these techniques are based on OFDM [1].

Despite the large benefits of OFDMA, The pronounced envelope fluctuations of the transmitted signal form a major problem especially in the uplink transmission. A large instantaneous swing of the transmitted signal power over the mean power level gets the signal distorted after the high power amplifier (HPA) at the transmitter end. Therefore, for zero distortion of the OFDMA signals, HPAs with a large dynamic range are required. These amplifiers are very expensive and complex, which place a significant burden on portable wireless terminals [2].

SC-FDMA [2] is proposed by 3GPP for uplink transmission in LTE/LTE-A. SC-FDMA is also known as Discrete Fourier Transform (DFT) spread OFDMA in which an extra DFT processing is added before OFDM modulation. Because of its inherent Single Carrier (SC) structure, SC-FDMA signals have a much smaller Peak to Average Power Ratio (PAPR) compared to those of Multi Carrier (MC) systems.
According to [3-6], SC transmission requires Pulse Shaping (PS) before transmission to limit the band of the transmitted signal and to mitigate the Inter Symbol Interference (ISI). Nevertheless, using PS to limit the frequency bandwidth (BW) enlarges the PAPR of the transmitted signal [5]. As a result, there is a trade-off between PAPR and BW reduction in conventional SC-FDMA.

Several methods are introduced for reducing PAPR in OFDMA systems, which can also be applied to SC-FDMA systems. Some of the most common methods applied are constellation Shaping (CS) [7], Clipping and Filtering (CF) [8], Partial Transmit Sequence (PTS) [9], Selective Mapping (SLM) [10], and Pulse Shaping (PS) [11, 12].

Since the overall PAPR at the transmitter is determined by the combination of the chosen modulation scheme and PS and the low PAPR advantage of SC-FDMA can be lost by conventional Raised Cosine (RC) PS [5, 6], research has been ongoing over the last several years aiming to design a PS filter with a proper time-limited waveform that limits SC-FDMA PAPR without degrading the system's performance, based on the knowledge that PS is an effective and simple process compared to other PAPR reduction methods [11].

In [12], a new family of modified RC filters, Convex and Concave, was formulated to reduce PAPR. these newly derived filters hold a new design parameter, giving an additional degree of freedom to minimize the PAPR for a given roll-off factor, but are characterized by complex impulse response expressions.

The main objectives of this paper are to: (1) Quick review the SC-FDMA system model and its different subcarrier mapping schemes. (2) Propose a new linear combination between RC [5] pulse and Parametric Exponential pulse (PEXP) [13] to reduce the SC-FDMA PAPR and compare the results with conventional RC pulse and other pulses introduced in [12].

The rest of this paper is organized as follows: Section 2 presents SC-FDMA transmission structure including its different subcarrier mapping methods and their different time domain structures. Section 3 presents PS for SC-FDMA while section 4 introduced the proposed new pulse and investigates its effectiveness. The PAPR results of SC-FDMA system using the new PS filter are introduced in section 5 and section 6 concludes the paper.

\section{SC-FDMA SYSTEM MODEL}

The block diagram presented in Figure 1 shows a typical SCFDMA uplink transmitter which has much in common with conventional OFDMA transmitter [4]. SC-FDMA can be regarded as DFT-spread OFDMA, where time domain data symbols are transformed to frequency domain by DFT before 
going through OFDM modulation using Inverse Fast Fourier Transform (IFFT). The orthogonality of users is maintained due to assigning different subcarriers in the frequency domain to different users.

SC-FDMA transmitter maps the data bits into a symbol sequence using some modulation technique, e.g. Phase Shift Keying (PSK) or Quadrature Amplitude Modulation (QAM) then groups the modulated symbols into complex vectors each containing $\mathrm{N}$ symbols.

These symbols are converted into frequency domain via $\mathrm{N}$ point DFT, Eq. (1), where $\mathrm{N}$ is selected as $\mathrm{N}=2^{\mathrm{b}}$ for some integer $b$ to allow radix-2 implementation of DFT. Each of the $\mathrm{N}$-DFT outputs are mapped onto $\mathrm{M}$ orthogonal subcarriers, where $\mathrm{M}$ must be larger than $\mathrm{N}$. If $\mathrm{N}$ equals $\mathrm{M} / \mathrm{Q}$ and all terminals transmit $\mathrm{N}$ symbols per block, the system can handle $\mathrm{Q}$ simultaneous transmissions without co-channel interference. Thus, Q can be referred to as the BW expansion factor of the symbol sequence.

$$
\widetilde{X}(k)=\sum_{n=0}^{N-1} x(n) e^{-j \frac{2 \pi}{N}} n k t, k=0,1, \ldots, N-1 .
$$

An M-point IFFT, Eq. (2), transforms the subcarrier amplitudes into a complex time domain samples. Before transmission, the signal vector is padded with Cyclic Prefix (CP). The $\mathrm{CP}$ is chosen to be slightly longer than the longest expected delay spread in the radio channel to be able to combat the ISI caused by SC modulation and multipath propagation.

$$
\tilde{\mathrm{x}}(\mathrm{v})=\frac{1}{\mathrm{M}} \sum_{\mathrm{m}=0}^{\mathrm{M}-1} \widetilde{\mathrm{X}}(\mathrm{m}) \mathrm{e}^{\mathrm{j} \frac{2 \pi}{\mathrm{M}} \mathrm{mv}}, \quad \mathrm{v}=0,1, \ldots, \mathrm{M}-1 .
$$

Since CP is a copy of the last part of the block, the discrete time linear convolution between the transmitted data and the channel can be converted into a discrete time circular convolution allowing for straightforward low complexity Frequency-Domain Equalization (FDE) at the receiver such as Zero Forcing (ZF) equalization [5]. The transmitter also performs an important linear filtering operation before transmission referred to as PS in order to reduce Out of Band (OoB) signal energy. RC is the most commonly used PS filter with SC-FDMA transmitter [6].

Two general subcarrier mapping schemes are introduced in [3] for allocating the subcarriers for SC-FDMA transmission: Distributed-FDMA (DFDMA) and Localized-FDMA (LFDMA).

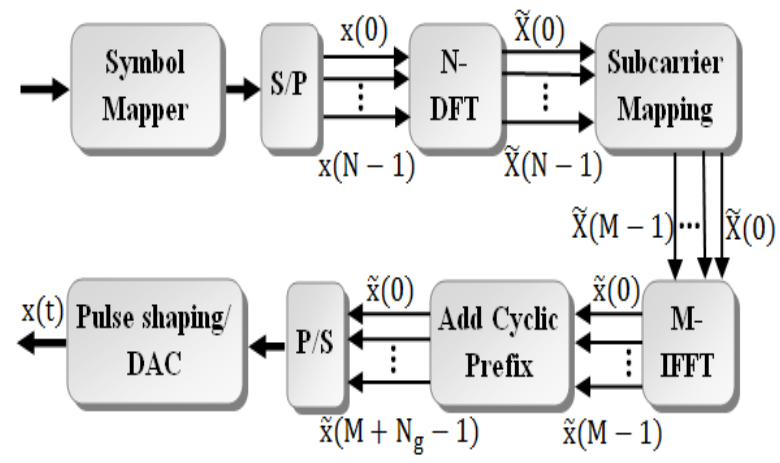

Fig 1: Block diagram of SC-FDMA transmitter.

In the Distributed-FDMA (DFDMA) subcarrier mapping, the DFT outputs are allocated over the entire bandwidth randomly with zeros occupying the unused subcarriers. Thus, DFDMA is robust against frequency selective fading due its built-in frequency diversity. A special case of the distributed mode with equidistance between occupied subcarriers is called Interleaved-FDMA (IFDMA)

On the other hand, In LFDMA subcarrier mapping, consecutive subcarriers are occupied by the DFT outputs while the rest of the BW is occupied by zeros. Even LFDMA can achieve multi-user diversity in frequency selective fading channel if each user is assigned a portion of the signal band that guarantees maximum channel gain [5].

Figure 2 illustrates an example for LFDMA and IFDMA subcarrier mapping schemes for a system of total subcarriers, $\mathrm{M}=8$, with four subcarriers, i.e. $\mathrm{N}=4$, assigned to each of the two simultaneous users, i.e. $\mathrm{Q}=2$.

\subsection{Time Domain Symbols of IFDMA:}

From the prosperities of FFT, Different allocation of zeros in the frequency domain would produce different signals in the time domain. Therefore, IFDMA and LFDMA are two SC systems with different time domain structures. These different time domain structures would differently affect their PAPR results.

In IFDMA, allocating zeros equally spaced in the frequency domain produces a repetition of the original modulated input symbols in time domain.

Since the frequency samples of IFDMA, $\widetilde{\mathrm{X}}_{\mathrm{I}}(\mathrm{m})$, are described as follows

$$
\widetilde{\mathrm{X}}_{\mathrm{I}}(\mathrm{m})=\left\{\begin{array}{cc}
\widetilde{\mathrm{X}}(\mathrm{k}), & \mathrm{m}=\mathrm{k} \cdot \mathrm{Q}\left(\begin{array}{c}
0 \leq \mathrm{k} \leq \mathrm{N}-1) \\
0,
\end{array},\right. \text { Otherwise }
\end{array},\right.
$$

where $0 \leq \mathrm{m} \leq \mathrm{M}-1$

Then, the time domain symbols, $\tilde{\mathrm{x}}_{\mathrm{I}}(\mathrm{v})$, derived in [3] for the interleaved mode can be obtained as follows

$$
\tilde{\mathrm{x}}_{\mathrm{I}}(\mathrm{v})=\operatorname{IFFT}\left(\widetilde{\mathrm{X}}_{\mathrm{I}}(\mathrm{m})\right)=\frac{1}{\mathrm{Q}} \mathrm{x}(\mathrm{n}),
$$

where $0 \leq n \leq N-1$ and $0 \leq v \leq M-1$. From Eq. (4), the resulting IFDMA time symbols, $\tilde{\mathrm{x}}_{\mathrm{I}}(\mathrm{v})$, consist of the original modulated symbols, $\mathrm{x}(\mathrm{n})$, repeated $\mathrm{Q}$ times and multiplied by a scaling factor equal to $1 / \mathrm{Q}$ as shown in Figure 3.

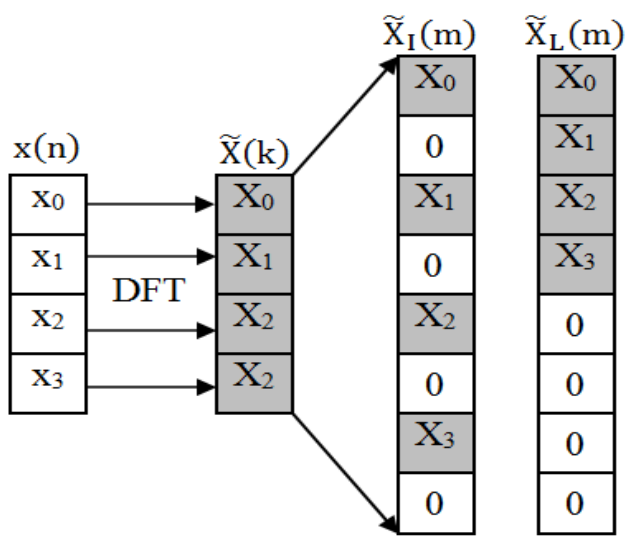

Fig 2: An example of SC-FDMA transmit symbols in the frequency domain for $\mathrm{N}=4$ subcarriers per user, $Q=2$ users, and $M=8$ subcarriers in the system.

Therefore, IFDMA is expected to have additional zero PAPR as a multiple access scheme and the effective PAPR is the one produced by the base band modulation scheme only. Therefore, PAPR of IFDMA is similar to conventional SC system. 


\subsection{Time Domain Symbols of LFDMA:}

On the other hand, in LFDMA, allocating data symbols subsequently in the frequency domain followed by zeros produces a repetition of the original input signal in certain positions in the time domain signal and a complex summation of the original modulated symbols in the rest of the time domain signal positions.

This was proven in [3] when the time domain signal of LFDMA, $\tilde{\mathrm{x}}_{\mathrm{L}}(\mathrm{v})$, was driven from the corresponding frequency samples, $\widetilde{X}_{\mathrm{L}}(\mathrm{m})$, as follows

$$
\widetilde{X}_{\mathrm{L}}(\mathrm{m})=\left\{\begin{array}{cc}
\widetilde{\mathrm{X}}(\mathrm{k}), & 0 \leq \mathrm{m} \leq \mathrm{N}-1 \\
0, & \mathrm{~N} \leq \mathrm{m} \leq \mathrm{M}-1
\end{array},\right.
$$

where $0 \leq \mathrm{k} \leq \mathrm{N}-1$ and $0 \leq \mathrm{m} \leq \mathrm{M}-1$.

Then,

$$
\tilde{x}_{L}(v)\left\{\begin{array}{ll}
\frac{1}{Q} x(n) & , q=0 \\
\frac{1}{Q}\left(1-e^{j 2 \pi \frac{q}{Q}}\right) \frac{1}{N} \sum_{n=0}^{N-1} \frac{x(n)}{1-e^{\left.\frac{j 2 \pi}{N}(z-n)+\frac{q}{Q}\right\}}}, q \neq 0
\end{array},\right.
$$

where $\mathrm{v}=(\mathrm{Q} \cdot \mathrm{z})+\mathrm{q}$ and $0 \leq \mathrm{z} \leq(\mathrm{N}-1), 0 \leq \mathrm{q} \leq(\mathrm{Q}-1)$.

Figure 4 and Eq. (6) illustrate that LFDMA time symbols have exact copies of input time symbols, $x(n)$, with a scaling factor of 1/Q in the sample positions that equal integer multiples of $\mathrm{Q}$ while the in-between samples contain a weighted sum of all the symbols in the input block, $x(n)$. As a result, it is expected that the resulting waveform will produce a high output peak which would increase PAPR for the LFDMA samples significantly [3].

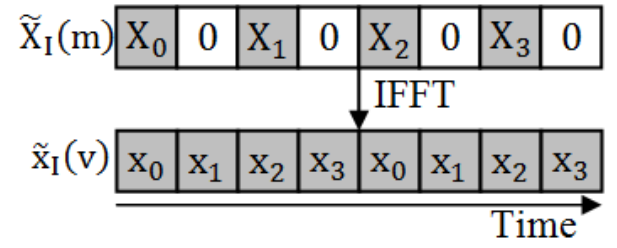

Fig 3: An example of IFDMA transmit symbols in the time domain for $N=4$ subcarriers per user, $Q=2$ users, and $M$ $=8$ subcarriers in the system.

$$
\begin{aligned}
& \begin{array}{l|l|l|l|l|l|l|l|l|}
\widetilde{\mathrm{X}}_{\mathrm{L}}(\mathrm{m}) & \mathrm{X}_{0} & \mathrm{X}_{1} & \mathrm{X}_{2} & \mathrm{X}_{3} & 0 & 0 & 0 & 0 \\
\hline \multicolumn{7}{|c|}{} \\
\hline
\end{array} \\
& \begin{array}{|l|l|l|l|l|l|l|l|}
\tilde{\mathrm{x}}_{\mathrm{L}}(\mathrm{v}) \\
\hline \mathrm{x}_{0} & *_{0} & \mathrm{x}_{1} & *_{1} & \mathrm{x}_{2} & *_{2} & \mathrm{x}_{3} & *_{3} \\
\hline
\end{array} \\
& *_{y}=\sum_{n=0}^{N-1} c_{q, z}(n) \cdot x(n), \quad y=0,1, \ldots,(M-N-1) \\
& , c_{q, z}(n)=\frac{1}{8}\left(\frac{1-e^{j \pi q}}{1-e^{\left.j \frac{\pi}{4}(z-n)+\frac{q}{2}\right\}}}\right): \text { Complex Weight }
\end{aligned}
$$

Fig 4: An example of LFDMA transmit symbols in the time domain for $N=4$ subcarriers per user, $Q=2$ users, and $M=8$ subcarriers in the system.

\section{PULSE SHAPING}

According to [4], PS is necessary for SC systems to band limit the transmitted signal and to mitigate ISI. Therefore, the selected pulse, $\mathrm{p}(\mathrm{t})$, must satisfy the following Nyquist criteria for zero ISI [11]

$$
p(t)=\left\{\begin{array}{lc}
1, & t=0 \\
0, & t= \pm T, \pm 2 T, \ldots
\end{array},\right.
$$

where $\mathrm{T}$ is the symbol period. The popular RC pulse, satisfies the zero ISI conditions, is widely used with SC systems. RC pulse can be considered as a sinc-shaped pulse multiplied by a damping factor and has the following impulse response [6].

$$
\mathrm{P}_{\mathrm{RC}}(\mathrm{t})=\operatorname{sinc}(\tau) \frac{\cos (\pi \alpha \tau)}{1-(2 \alpha \tau)^{2}}, 0 \leq \alpha \leq 1,
$$

where $\tau$ is the normalized time, $\tau=t / T$ and $\alpha$ is the roll-off factor. This factor $\alpha$ is very important as it determines the BW occupied by the pulse, BW $=(1+\alpha) / 2 \mathrm{~T}$. $\alpha$ also determines the rate at which the tails of the pulse decay. $\alpha$ varies according to the implemented technology. For example, in wireless communication systems, $\alpha$ is in the range of $0.22 \leq \alpha$ $\leq 0.5[10]$.

In $[3,5,6]$, RC PS was found harmful for SC-FDMA in terms of PAPR, especially for IFDMA while the PAPR of LFDMA hardly increased. This is due to their different time domain structures as mentioned earlier. As a result, there is a trade-off between spectrum efficiency and PAPR reduction in conventional SC systems.

Since much of IFDMA advantages are lost by conventional RC PS, it became necessary to design a new PS filter that would not degrade the system performance and reduce the PAPR at the same time.

That is why a newly developed PS filter is proposed to reduce IFDMA PAPR while maintaining the same excess BW as RC filter. For keeping the zero ISI condition, the new PS filter must also satisfy the Nyquist-I criterion. Moreover, it has to be simple, unlike other existing pulses [13], and has flexible mathematical expression as possible in order to reduce the processing time of the system, which in advance reducing the cost

\section{PROPOSED NEW PULSE SHAPING}

As mentioned in [11], Nyquist pulses have different decay rates, that decay rate has great impact on PAPR. In order to minimize the PAPR of the modulated signal, it is better to design a pulse with a reduced tail size as the relative magnitudes of the two largest side lobes of the filter response in the time domain are the ones that have the biggest impact on PAPR.

\subsection{Basic Idea}

According to [11], a linear combination between two Nyquist pulses ensures achieving the Nyquist criteria providing distortionless transmission. Therefore, a linear combination between the conventional RC pulse [6] and PEXP [13] is considered in this paper to reduce PAPR of IFDMA. RC and PEXP are pulses that have an explicit time-domain functions having decay rates of $1 / \mathrm{t}^{3}$ and $1 / \mathrm{t}^{2}$, respectively.

The impulse response of the PEXP can be expressed as follows [13]

$$
\begin{gathered}
P_{P E X P}(t)=\operatorname{sinc}(\tau) \cdot m(t), \\
m(t)=\frac{2\{(\pi \alpha) /(\ln 2)\} \tau \sin (\pi \alpha \tau)+2 \cos (\pi \alpha \tau)-1}{((\pi \alpha) /(\ln 2) \tau)^{2}+1} .
\end{gathered}
$$

Since the linear combination is simply a process of vector addition and scalar multiplication [14], then, the proposed 
New Nyquist Linear Combination Pulse (NNLCP) can be defined as follows

$$
P_{N N L C P}(t)=C \cdot P_{P E X P}(t)+B \cdot P_{R C}(t),
$$

Where $\mathrm{C}$ and $\mathrm{B}$ are linear combination constants which are defined for all real numbers. But in order to comply with Nyquist first criterion (in the time-domain) [15], B must be equal to $(1-C)$. The constant $C$ adds an additional degree of freedom to minimize PAPR for a given $\alpha$.

Substituting by Eqns. (8) and (9) into Eq. (11), the impulse response of the NNLCP can be obtained as

$$
P_{N N L C P}(t)=\operatorname{sinc}(\tau)\left[C \cdot m(t)+(1-C) \cdot \frac{\cos (\pi \alpha \tau)}{1-(2 \alpha \tau)^{2}}\right] .
$$

\subsection{Frequency and Impulse Response:}

In Figure 5, the frequency response of the NNLCP with different values of $\mathrm{C}$ is compared with conventional $\mathrm{RC}$ pulse for $\alpha$ equal to 0.35 , which is a roll-off factor commonly used in literature. In general, it is illustrated that the new pulse has the same BW compared to that of the RC filter with different values of $\mathrm{C}$. Therefore, the new pulse will not introduce additional OoB radiation into the transmitted signal, which is a major concern in the design of PS filters for PAPR reduction.

On the other hand, Figure 6 illustrates the impulse response of the conventional RC filter with an excess bandwidth, $\alpha=$ 0.35 , compared to NNLCP with different values of C. It is obvious that the RC filter has larger side lobes than those of the NNLCP which in turn have a large impact on PAPR as will be confirmed by PAPR simulations later. Therefore, selecting the proper filters based on their decay rate is a priority when trying to minimize PAPR.

Further, we can see from Figure 6 that the proposed pulse equals one when $t=0$ for different values of C. Additionally, the proposed pulse always equals zero when evaluated for $\mathrm{t} / \mathrm{T}= \pm 1, \pm 2, \ldots$ and different values of $\mathrm{C}$. Therefore, the ISIfree criterion holds for the proposed new pulse.

\section{PERFORMANCE EVALUATION}

Regarding the impulse response of the NNLCP for different values of $\mathrm{C}$, the $\mathrm{RC}$ filter has larger side lobes than those of the proposed filter. Thus, by selecting the proper $\mathrm{C}$, a reduction in the relative amplitudes of the two largest side lobes can be accomplished which would lead to PAPR reduction.

The optimal value of the linear combination constant, C, differs for every roll-off factor and SC transmission scheme, although it might not be unique. To determine this optimal value for PAPR reduction, extensive computer simulations are performed because an analytical solution seems unrealizable as the statistical properties of PAPR for SC modulations are not easily obtained analytically.

Since $\mathrm{C}$ is defined for all real numbers and to limit these probabilities, let the case for $|\mathrm{C}| \gg 1$ be considered first before the numerical analysis. Then, $\mathrm{Eq}(12)$ turns to the form

$$
P_{N N L C P}(t)=C\left[P_{P E X P}(t)-P_{R C}(t)\right]
$$

From Eq. (13), it seems that the larger the value of $C$, the larger will be the side loops of $\mathrm{P}_{\mathrm{NNLCP}}(\mathrm{t})$, which would increase the PAPR significantly. Therefore, it is unlikely that the PAPR has a minimum value for $|C| \gg 1$, as will be proven by simulations later. Moreover, in [15], it was also proven that it is unlikely to have minimum timing errors at the same condition.

To evaluate the PAPR of individual system configurations, Table 1 represents the simulation parameters used for the PAPR analysis based on LTE-A standard [16]. PAPR is statistically evaluated by using complementary cumulative distribution function (CCDF) which is the probability that PAPR is greater than a PAPR value $\mathrm{PAPR}_{0}(\operatorname{Pr}\{\mathrm{PAPR}>$ $\left.\left.\mathrm{PAPR}_{\mathrm{o}}\right\}\right)$.

Results are observed at $\mathrm{PAPR}_{\mathrm{o}}$ value that is exceeded with probability less than $0.1 \%\left(\operatorname{Pr}\left\{\mathrm{PAPR}>\mathrm{PAPR}_{\mathrm{o}}\right\}=10^{-3}\right)$, or 99.9 percentile PAPR.

Figure 7 and Figure 8 illustrate the numerical analysis of PAPR performance for IFDMA and LFDMA systems, respectively, using the new filter with different values of $\mathrm{C}$. To be clear, only PAPR curves evaluated with positive values of $\mathrm{C}$ are plotted due to lack of space, but the case will be the same for negative values of $\mathrm{C}$.

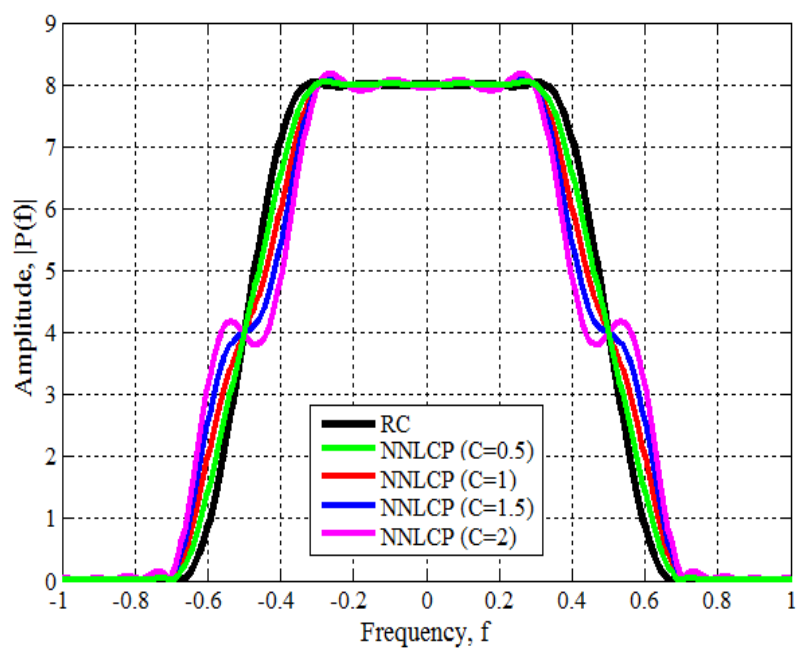

Fig 5: Frequency response of the RC filter and NNLCP for different values of $C$ and $\alpha=0.35$.

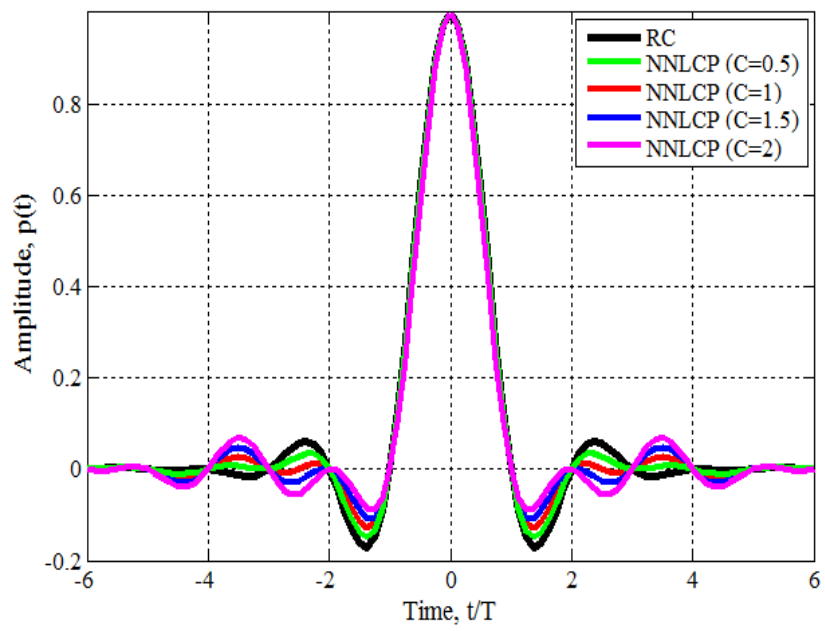

Fig 6: Impulse response of the RC filter and New Nyquist Linear Combination Pulse (NNLCP) for different values of $C$ and $\alpha=0.35$. 
Table 1. Default Values for System Parameters

\begin{tabular}{|c|c|c|}
\hline Channel bandwidth & BW & $5 \mathrm{MHz}$ \\
\hline System Subcarriers & $\mathrm{M}$ & 256 \\
\hline User Subcarriers & $\mathrm{N}$ & 64 \\
\hline BW Spreading Factor & $\mathrm{Q}$ & 4 \\
\hline $\begin{array}{c}\text { Modulation } \\
\text { Uniform Random Data } \\
\text { bits }\end{array}$ & $16-\mathrm{QAM}$ \\
\hline $\begin{array}{c}\text { Subcarrier Mapping } \\
\text { CCDF clip rate }\end{array}$ & \multicolumn{2}{|c|}{$10^{5}$} \\
\hline $\begin{array}{c}\text { Pulse Shaping Filters } \\
\text { Baseband Pulses p(t) } \\
\text { Truncated From }\end{array}$ & $-6 \mathrm{~T}$ to $+6 \mathrm{~T}$ time periods \\
\hline $\begin{array}{c}\text { Oversampling } \\
\text { RNCP }\end{array}$ \\
\hline
\end{tabular}

From Figure 7 and Figure 8, it is observed that for $|\mathrm{C}| \gg 1$, the PAPR of both systems significantly increase, as expected. That is because, for $|C| \gg 1$, the larger the value of $C$, the larger will be the side lobes as shown by Figure 6. According to these numerical analysis, the optimal values of $C$ are equal to 1.5 and 0.6 for IFDMA and LFDMA, respectively, with $\alpha$ equal to 0.35 .

Referring to Figure 9, the effectiveness of the optimum new PS filter with IFDMA is demonstrated. Simulations proved that the proposed NNLCP filter achieves PAPR results exceeding those of conventional RC pulses. Moreover, NNLCP has lower PAPR compared to Convex and Concave pulses proposed in [12] with simpler impulse response.

On the other hand, the performance of the proposed new PS filter, NNLCP, with LFDMA is shown in Figure 10. LFDMA did not show PAPR improvement with the new pulse compared to RC, the same result is observed with other pulses proposed for comparison [12].

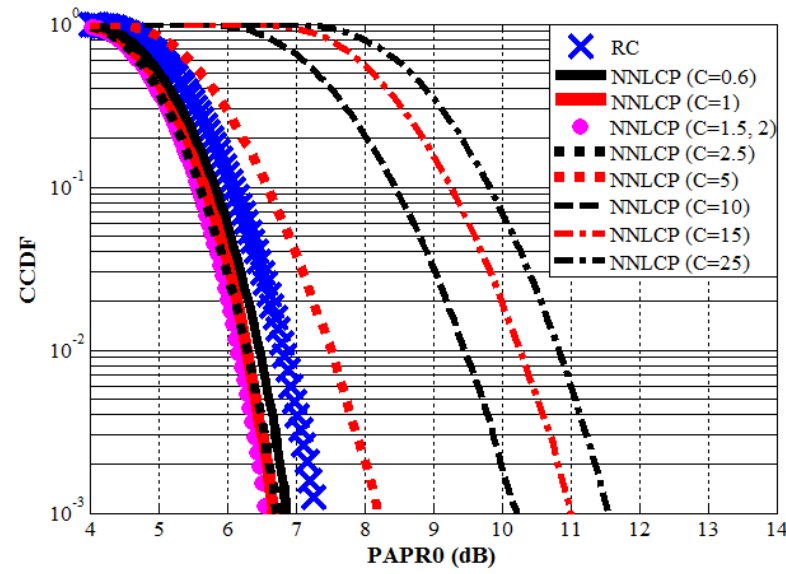

Fig 7: CCDF of PAPR for IFDMA system with RC filter and NNLCP for different values of $C$ and $\alpha=0.35$.

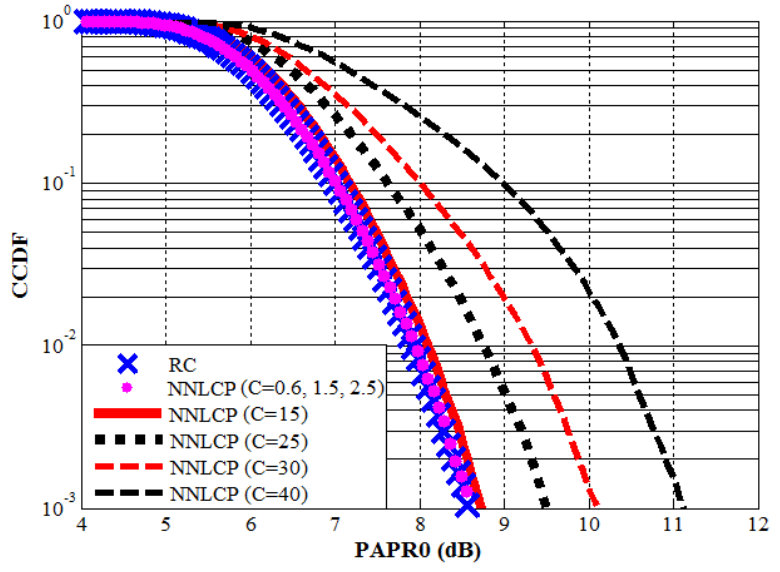

Fig 8: CCDF of PAPR for LFDMA system with RC filter and NNLCP for different values of $C$ and $\alpha=0.35$.

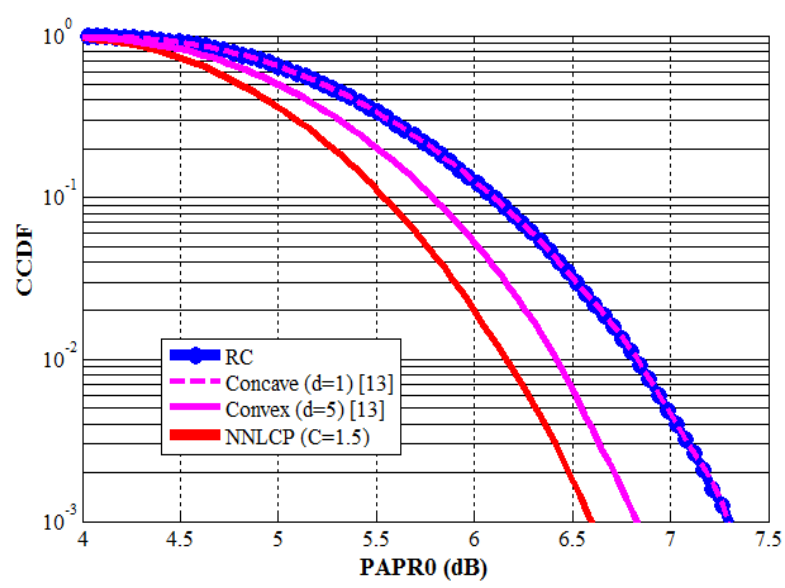

Fig 9: CCDF of PAPR for IFDMA system using optimum NNLCP with $\alpha=0.35$.

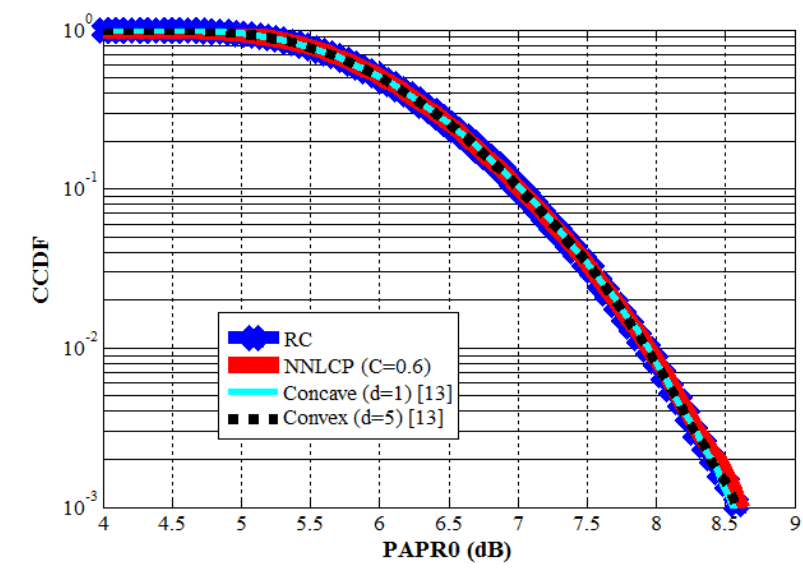

Fig 10: CCDF of PAPR for LFDMA system using optimum NNLCP with $\alpha=0.35$.

\section{CONCLUSIONS}

In this paper, a brief study of SC-FDMA proposed for LTE-A as the uplink radio access system was presented including its two subcarrier mapping schemes, LFDMA and IFDMA. This study was concerned with the PAPR since it is the main challenge facing any uplink transmission. Since the low IFDMA PAPR advantage can be lost by RC pulse shaping, a linear combination between the conventional RC pulse and parametric exponential pulse was proposed for PAPR reduction in IFDMA. The new pulse was found effective in 
reducing IFDMA PAPR via introducing an additional combination constant, $\mathrm{C}$, which provides a degree of freedom to minimize PAPR for a given roll-off factor. Simulations were conducted for determining the optimum value of $\mathrm{C}$ for IFDMA and LFDMA and were found equal to 1.5 and 0.6, respectively. Simulations also showed that the optimum proposed filter achieved better PAPR compared to RC filter in addition to other filters proposed for comparison in case of IFDMA while not significantly affecting LFDMA PAPR.

\section{FUTURE WORK}

The linear combination technique can be applied to any pair of pulses to obtain new useful pulses with lower PAPR. Therefore, the combination method can be applicable even to pulses that may be discovered in the future.

\section{REFERENCES}

[1] E. Dahlman, S. Parkvall, J. Sköld, and P. Beming. 2008 3G Evolution: HSPA and LTE for Mobile Broadband, USA. Academic Press. 2nd ed.

[2] S. Litsyn. 2007 Peak Power Control in Multicarrier Communications. Cambridge University Press. 2nd ed.

[3] H. G. Myung, J. Lim, and D. J. Goodman. 2008 Single Carrier FDMA A New Air Interface for Long Term Evolution. U.K. Wiley, 1st ed.

[4] H. G. Myung. 2007. Introduction to Single Carrier FDMA. 15th European Signal Processing Conference (EUSIPCO 2007), Poland.

[5] H. G. Myung, J. Lim, and D. J. Goodman, "Single Carrier FDMA for Uplink Wireless Transmission", IEEE, Vehicular Technology Mag.; 2006.

[6] H. G. Myung, J. Lim, and D. J. Goodman. 2006. PeakTo-Average Power Ratio of Single Carrier FDMA Signals With Pulse Shaping. The 17th Annual IEEE International Symposium on Personal, Indoor and Mobile Radio Comm. (PIMRC), Finland.

[7] M. Niranjan, and S. Srikanth. 2011. Adaptive Active Constellation Extension for PAPR Reduction in OFDM
Systems. IEEE International Conference on Recent Trends in Information Technology (ICRTIT), India.

[8] B. M. Lee, and Y. Kim, "An Adaptive Clipping and Filtering Technique for PAPR Reduction of OFDM Signals", Springer, Circuits, Syst.; and Signal Processing, 2013.

[9] J. Lain, S. Wu, and P. Yang, "PAPR Reduction of OFDM Signals Using PTS: A Real-valued Genetic Approach", Springer, EURASIP Journal on Wireless Comm. and Networking, 2011.

[10] K. Kim, H. Jeon, J. No, and D. Shin, "Low-complexity Selected Mapping Scheme Using Cyclic-shifted Inverse Fast Fourier Transform for Peak-to-Average Power Ratio Reduction in Orthogonal Frequency Division Multiplexing Systems", IEEE, Comm.; IET, 2013.

[11] C. A. Azurdia-Meza. 2013 Analysis, Design, and Implementation of Parametric Nyquist ISI-Free Pulses in Wireless Communications Systems. Doctoral Thesis. Dept. of Electronics and Radio Eng., Graduate School Kyung Hee Univ., Korea.

[12] S. Mohapatra, S. Das. 2009. Peak-to-Average power Reduction (PAPR) by Pulse Shaping Using a Modified Raised Cosine Filters. Annual IEEE India Conference (INDICON), Gujarat.

[13] C. A. Azurdia-Meza. 2013. PAPR Reduction in SCIFDMA Uplink System Using Parametric Pulses. IEEE, Santiago.

[14] G. Strang, 1998 Introduction to Linear Algebra. Wellesley-Cambridge Press,

[15] P. Sandeep, S. Chandan, and A. K. Chaturvedi, "ISI-Free Pulses With Reduction Sensitivity to Timing Errors," IEEE Comm. Lett., Apr. 2005.

[16] A. Ghosh, R. Ratasuk, B. Mondal, N. Mangalvedhe, T. Thomas, "LTE-advanced: Next-generation Wireless Broadband Technology", [Invited Paper], IEEE ,Wireless Comm. 2010. 